02,05

\title{
Универсальность поведения гистерезиса магнитосопротивления и его температурной эволюции для гранулярных высокотемпературных сверхпроводников $\mathrm{Y}-\mathrm{Ba}-\mathrm{Cu}-\mathrm{O}$
}

\author{
(C) С.В. Семенов ${ }^{1,2}$, Д.А. Балаев ${ }^{1,2}$, М.И. Петров ${ }^{1}$ \\ ${ }^{1}$ Институт фризики им. Л.В. Киренского ФИЦ КНЦ СО РАН, \\ Красноярск, Россия \\ ${ }^{2}$ Сибирский фредеральный университет, \\ Красноярск, Россия \\ E-mail: svsemenov@iph.krasn.ru
}

Поступила в Редакцию 17 марта 2021 г.

В окончательной редакции 17 марта 2021 г.

Принята к публикации 22 марта 2021 г.

\begin{abstract}
Посвящена установлению закономерности в поведении гистерезиса магнитосопротивления $R(H)$ гранулярных высокотемпературных сверхпроводников (ВТСП) иттриевой системы. Для этого проведено сравнительное исследование магнитотранспортных свойств образцов гранулярных ВТСП, обладающих (i) примерно одинаковыми магнитными свойствами и температурами начала сверхпроводящего перехода $(90.5-93.5 \mathrm{~K}$, что характеризует ВТСП гранулы) и (ii) различной величиной транспортного критического тока $J_{\mathrm{C}}$ (что характеризует межгранульные границы). Несмотря на значительный разброс в величинах $J_{\mathrm{C}}$ (более чем на порядок) исследованных трех образцов, обнаружено универсальное поведение гистерезиса магнитосопротивления, видимо, присущее всем гранулярным $\mathrm{Y}-\mathrm{Ba}-\mathrm{Cu}-\mathrm{O}$. Гистерезис $R(H)$ очень широкий, и в достаточно большом интервале внешнего поля зависимость ширины гистерезиса магнитосопротивления $\Delta H$ от поля $H_{\mathrm{dec}}$ (внешнее поле $H=H_{\mathrm{dec}}$ для убывающей ветки гистерезиса) близка к линейной функции: $\Delta H \approx H_{\text {dec. }}$ Это поведение наблюдается для всей температурной области реализации сверхпроводящего состояния (исследования проведены при температурах 77-88 и 4.2 K). Объяснение полученному результату основывается на концепции рассмотрения эффективного поля в межгранульных границах, которое является суперпозицией внешнего поля и поля, индуцированного магнитными моментами гранул. Индуцированное гранулами поле, в свою очередь, значительно усиливается в области межгранульных границ, благодаря эффекту сжатия магнитного потока (протяженность межгранульных границ на несколько порядков меньше размера ВТСП гранул). Вышесказанное подтверждается анализом гистерезиса $R(H)$ для композитного ВТСП на основе $\mathrm{Y}-\mathrm{Ba}-\mathrm{Cu}-\mathrm{O}$ и $\mathrm{CuO}$, в котором протяженность межгранульных границ целенаправленно увеличена, как следствие, влияние эффекта сжатия потока проявляется меньше, а гистерезис $R(H)$ сужается.
\end{abstract}

Ключевые слова: гранулярные ВТСП, гистерезис магнитосопротивления, межгранульные границы.

DOI: 10.21883/FTT.2021.07.51034.055

\section{1. Введение}

Хорошо известно, что поликристаллические (далее - гранулярные) высокотемпературные сверхпроводники (ВТСП) по своим, транспортным и магнитотранспортным свойствам представляют собой двухуровневую сверхпроводящую систему. Для ВТСП гранул характерны достаточно большие плотности критического тока (так называемый внутригранульный критический ток $J_{\mathrm{CM}}$ ), и именно внутригранульные токи определяют магнитный отклик „объемных“ образцов в умеренных и сильных магнитных полях. В то же время, наличие межгранульных границ, имеющих толщину всего не более нескольких нанометров, уже существенно (на несколько порядков) уменьшает критическую плотность транспортного тока „объемных“ образцов. Это происходит из-за того, что толщина, или геометрическая протяженность межгранульных границ имеет порядок длины когерентности ВТСП, и в результате перенос сверхпроводящего тока через межгранульные границы происходит посредством джозефсоновского туннелирования, при котором критический ток значительно меньше. Вышесказанное можно считать основой применимости концепции двухуровневой (гранулы и границы) сверхпроводящей системы [1] к описанию магнитных и транспортных свойств гранулярных ВТСП. При этом очевидно строгое неравенство $J_{\mathrm{CM}} \gg J_{\mathrm{C}}$, где $J_{\mathrm{C}}-$ транспортный критический ток. За немалое время, прошедшее после открытия ВТСП материалов, были определены многие закономерности во влиянии различных факторов на морфологию межгранульных границ, и, в конечном счете, на поведение транспортных характеристик [2-10]. Для описания процессов диссипации в подсистеме межгранульных границ можно применять стандартные подходы, развитые для сверхпроводников II рода [10-23]. Отметим, что, несмотря на большое количество работ, посвященных описанию процессов диссипации в гранулярных ВТСП системах [10-24], 
недавно были обнаружены особенности поведения электросопротивления во внешних полях, указывающие на протекание топологического фазового перехода - перехода Березинского-Костерлица-Таулеса в межгранульной среде [25-28].

Однако, если рассматривать уже магнитотранспортные характеристики гранулярных ВТСП (магнитосопротивление $R(H)$, транспортный критический ток $J_{\mathrm{C}}(H)$ в зависимости от внешнего поля), то для понимания большинства эффектов уже необходимо принимать во внимание взаимодействие указанных сверхпроводящих подсистем. Действительно, есть совокупность экспериментальных фактов, которые невозможно объяснить в рамках обычного подхода. К таким фактам можно отнести следующие:

1) достаточно сложная форма гистерезиса $R(H) \quad[29-42] \quad$ и $\quad J_{\mathrm{C}}(H) \quad[43-47], \quad$ при $\quad$ этом $R\left(H_{\mathrm{inc}}\right)>R\left(H_{\mathrm{dec}}\right)$ и $J_{\mathrm{C}}\left(H_{\mathrm{inc}}\right)<J_{\mathrm{C}}\left(H_{\mathrm{dec}}\right)$ при циклировании внешнего поля до некоторой постоянной величины $\pm H_{\max }$ (здесь и далее индексы ,inc“ и „dec“ относятся к возрастающему и убывающему внешнему полю соответственно); хотя для начальной ветки магнитосопротивления $R\left(H_{\text {ini }}\right)$ при относительно небольших значениях внешнего поля имеет место обратная ситуация: $R\left(H_{\mathrm{ini}}\right)<R\left(H_{\mathrm{dec}}\right)$.

2 ) немонотонное поведение зависимостей $R\left(H_{\text {inc }}\right)$ (достаточно часто наблюдается локальный максимум) и $H_{\mathrm{dec}}$ (есть минимум, либо сопротивление становится равным „нулю“ $)$ [29,31-33,36-39,42].

3) анизотропия магнитосопротивления относительно взаимной ориентации внешнего поля и макроскопического транспортного тока $\mathbf{j}$, при этом $R(\mathbf{H} \| \mathbf{j})<R(\mathbf{H} \perp \mathbf{j}) \quad[48-52,36,37]$, и гистерезис $R(H)$ шире для ,перпендикулярной“ $\mathbf{H} \perp \mathbf{j}$ ориентации [53-55].

4) влияние термомагнитной предыстории на зависимости $R(T)$ : условия охлаждения во внешнем поле $H$ приводят к меньшей диссипации, чем условия охлаждения без поля с последующим приложением внешнего поля $H$ той же величины [48,49,46,56-62,54].

5) весьма широкий гистерезис магнитосопротивления, т. е., большое значение величины $\Delta H$, определяемой как $\Delta H=H_{\mathrm{dec}}-H_{\mathrm{inc}}$ при условии $R\left(H_{\mathrm{dec}}\right)=R\left(H_{\mathrm{inc}}\right)$.

Совокупность перечисленных фактов (1)-(5) качественно объясняются в рамках концепции рассмотрения эффективного поля в межгранульной среде [63,64]. Здесь принципиальным является взаимодействие подсистемы гранул с подсистемой межгранульных границ, в котором магнитные моменты ВТСП гранул $\mathbf{M}_{\mathrm{G}}$ „наводят поле в межгранульных границах, см. рис. 1. В результате подсистема межгранульных границ находится в эффективном

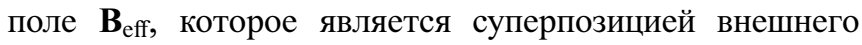
поля $\mathbf{H}$ и поля $\mathbf{B}_{\text {ind }}$, индуцированного гранулами в межгранульную среду. Магнитный отклик ВТСП гранул определяется и мейсснеровскими токами (в модели критического состояния циркулирует ток, чья плотность является критической), и абрикосовскими вихрями, захваченными гранулами. С учетом экспериментально наблю-

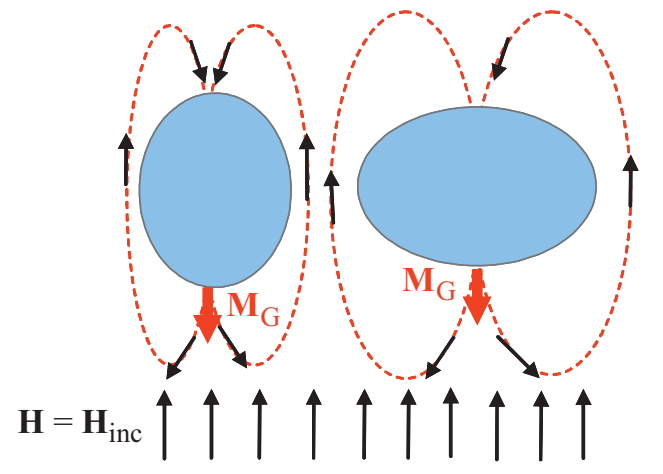

$a$

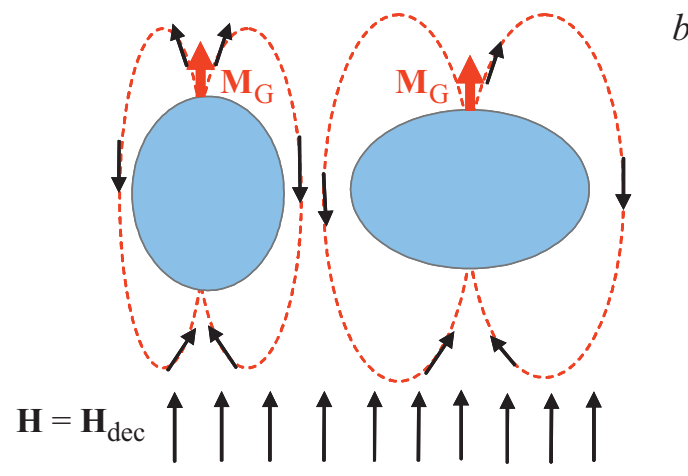

Pис. 1. Схематическое представление направлений линий магнитной индукции $\mathbf{B}_{\text {ind }}$ от магнитных моментов $\mathbf{M}_{\mathrm{G}}$ (направление $\mathbf{M}_{\mathrm{G}}$ определяется знаком намагниченности $M$ образца) ВТСП гранул (овалы) в межгранульной среде (пространство между гранулами в данном представлении сильно увеличено). Случаи $(a)$ и $(b)$ соответствуют возрастающему $\left(\mathbf{H}=\mathbf{H}_{\mathrm{inc}}\right)$ и убывающему $\left(\mathbf{H}=\mathbf{H}_{\mathrm{dec}}\right)$ полю. Для случая $(a) \mathbf{B}_{\text {ind }} \| \mathbf{H}$, a для случая $(b)$ линии магнитной индукции направлены против внешнего поля.

даемой гистерезисной зависимости намагниченности от поля $M(H)$ и направлением индуцированного поля $\mathbf{B}_{\mathrm{ind}}$, см. рис. 1, для эффективного поля в межгранульной среде справедливо следующее выражение:

$$
B_{\mathrm{eff}}(H)=|H-4 \pi M(H) \cdot \alpha| .
$$

Модуль в выражении (1) взят ввиду того, что для описания процессов диссипации не важен знак результирующего эффективного поля, и магнитосопротивление $R$ является функцией $B_{\text {eff: }} R=f\left(B_{\text {eff }}\right)$. Множитель $\alpha$ в выражении (1) включает в себя усредненный размагничивающий фактор, а также степень сжатия потока в промежутках между гранулами. Описание экспериментальных данных по гистерезису магнитосопротивления $R(H)$ гранулярных ВТСП по выражению (1) дало хорошее качественное [65-69,54,55] и количественное [70] согласие. Однако такое согласие достигается, если только принять, что параметр $\alpha$ имеет значение порядка 20-25 (для „перпендикулярной“ $\mathbf{H} \perp \mathbf{j}$ ориентации). Это является доказательством сильного сжатия потока в межгранульной среде, что является следствием малой протяженности межгранульных границ в сравнении с 
размером ВТСП гранул (микрометры); впервые на возможность реализации такого сжатия магнитного потока было указано в работе [52].

В общепринятом подходе к описанию процессов диссипации сверхпроводников II рода зависимость магнитосопротивления от внешнего поля $H$, температуры $T$, и транспортного тока $I$ определяется зависимостью от этих параметров потенциала пиннинга $U_{\mathrm{P}}(H, T, I)$ через выражение аррениусовского типа $R(H, T, I)=R_{\mathrm{N}} \exp \left(-U_{\mathrm{P}}(H, T, I) / k T\right) \quad[71], \quad$ в котором $R_{\mathrm{N}}$ - сопротивление в „нормальном“ состоянии. Для межгранульных границ, или джозефсоновской среды, вместо $U_{\mathrm{P}}(H, T, I)$, логично использовать термин „энергия джозефсоновской связи“ $E_{\mathrm{J}}(H, T, I)$, а ввиду выражения (1), вместо внешнего поля $H$, использовать уже эффективное поле $B_{\text {eff }}(H)$ [70]:

$$
\begin{aligned}
R(H, T, I) & =R\left(B_{\mathrm{eff}}(H), T, I\right) \\
& =R_{\mathrm{N}} \exp \left(-E_{\mathrm{J}}\left(B_{\mathrm{eff}}(H), T, I\right) / k T\right) .
\end{aligned}
$$

Ясно, что зависимость $E_{\mathrm{J}}\left(B_{\mathrm{eff}}(H), T, I\right)$ определяется многими факторами, такими как средняя толщина межгранульных границ, их тип (металл, диэлектрик), и др. Ясно, что эти факторы зависят от условий синтеза и окончательной термообработки ВТСП материалов, и здесь логично ожидать совершенно различное поведение магнитосопротивления и его температурную эволюцию для ВТСП образцов, приготовленных различными методиками. Однако в данной работе мы показываем, что есть универсальный характер температурной эволюции гистерезиса магнитосопротивления, одинаковый для образцов с различной токонесущей способностью. Это демонстрируется на примере системы $\mathrm{YBa}_{2} \mathrm{Cu}_{3} \mathrm{O}_{7}$ для трех образцов с различными условиями приготовления и критическим током. Указанный „универсальный характер“ поведения уже не наблюдается для композитного ВТСП, в котором добавление несверхпроводящего компонента дополнительно уменьшает энергию джозефсоновской связи (и, следовательно, критический ток) ввиду увеличения протяженности межгранульных границ. Полученные результаты имеют тесную связь с эффектом сжатия магнитного потока в межгранульной среде.

\section{2. Эксперимент}

Исследованные образцы ВТСП $\mathrm{YBa}_{2} \mathrm{Cu}_{3} \mathrm{O}_{7}$ приготавливались методом твердофазного синтеза с 3-5 промежуточными помолами. Для образца с наибольшей плотностью критического тока (образец ҮВСО-1) окончательная стадия отжига проводилась при температуpe около $940^{\circ} \mathrm{C}$ (близкой к температуре плавления) в течение $50 \mathrm{~h}$. По окончании синтеза образец был отожжен при температуре $350^{\circ} \mathrm{C}$ в течение $10 \mathrm{~h}$ для достижения стехиометрии по кислороду. Для образцов YBCO-2 и YВСО-3 полное время отжига составляло
$160 \mathrm{~h}$ при температурах $920-930^{\circ} \mathrm{C}$; в конце синтеза образцы были отожжены при температуре $300^{\circ} \mathrm{C}$ в течение 3 h и медленно охлаждены вместе с печью до комнатной температуры для насыщения кислородом. По данным рентгенофазового анализа все пики полученных образцов принадлежат структуре $1-2-3$, посторонних рефлексов замечено не было.

Композитный образец (далее YВСО-comp), содержащий 77.5 Vol.\% сверхпроводника $\mathrm{Y}_{0.75} \mathrm{Lu}_{0.25} \mathrm{Ba}_{2} \mathrm{Cu}_{3} \mathrm{O}_{7}$ и $22.5 \mathrm{Vol} \%$ оксида меди $\mathrm{CuO}$, приготавливался методом быстрого спекания [72,53]. В этом методе использовался заранее приготовленный технологией твердофазного синтеза и охарактеризованный ВТСП $\mathrm{Y}_{0.75} \mathrm{Lu}_{0.25} \mathrm{Ba}_{2} \mathrm{Cu}_{3} \mathrm{O}_{7}$, к перемолотому порошку которого добавлялся в необходимой пропорции ОСЧ реактив $\mathrm{CuO}$. После совместного перетирания ингредиентов и прессования, полученная таблетка помещалась в печь, предварительно нагретую до $915^{\circ} \mathrm{C}$ на $5 \mathrm{~min}$, после чего образец перемещался в другую печь, нагретую до $350^{\circ} \mathrm{C}$, где в течении $4 \mathrm{~h}$ проходил отжиг с последующим остыванием в печи (для насыщения кислородом). По данным рентгенофазового анализа все пики полученного композитного образца принадлежат структуре $1-2-3$ и оксиду меди, посторонних рефлексов замечено не было.

Таблица содержит данные по физической плотности (определялась взвешиванием образцов в спирте) и среднему размеру гранул сверхпроводника (определялся из микрофотографий, полученных сканирующей электронной микроскопией на приборе Hitachi-TM 3000).

Магнитотранспортные измерения проводились четырехзондовым методом. Из таблеток выпиливались образцы размерами $\sim 0.15 \div 0.15 \div 7 \mathrm{~mm}^{3}( \pm 50 \%)$, транспортный ток $I$ прикладывался вдоль длинной оси образца. Использовались прижимные золоченые контакты. Критический ток определялся по критерию $1 \mu \mathrm{V} / \mathrm{cm}$, и при этом образец был погружен в среду жидкого азота или жидкого гелия. Внешнее поле задавалось электромагнитом при измерениях магнитосопротивления $R(H)$ в высоких температурах (от $77 \mathrm{~K}$ ), и сверхпроводящим соленоидом в измерениях $R(H)$ при $T=4.2 \mathrm{~K}$. Зависимости $R(H)$ при температурах $4.2 \mathrm{~K}$ и $77 \mathrm{~K}$ для образцов ҮВСО, измеренные при транспортном токе $\sim 30 \mathrm{~mA}$ и более, были получены при непосредственном помещении образца в жидкий гелий, либо в жидкий азот соответственно (во избежание разогрева на токовых контактах). При измерениях зависимостей $R(T)$ и $R(H)$ при малых значениях измерительного тока (до $10 \mathrm{~mA}$ ) образец находился в гелиевой теплообменной среде. Часть измерений $R(T)$ и $R(H)$ была проведена на установке PPMS-6000. Во всех случаях использовался режим охлаждения образца в нулевом внешнем поле, а прикладываемое внешнее поле было перпендикулярно направлению тока $\mathbf{H} \perp \mathbf{I}$.

Магнитные свойства исследованы на вибрационном магнетометре при внешних условиях (включая скорость развертки внешнего магнитного поля), соответствующих магнитотранспортным измерениям. 
Значения транспортного $J_{\mathrm{C}}$ критического тока, удельного сопротивления, $\rho$, внутригранульного („магнитного“) критического тока $J_{\mathrm{CM}}$ (выражение $(3)$ ), среднего размера гранул $\langle d\rangle$ и физической плотности исследованных образцов

\begin{tabular}{c|c|c|c|c|c|c|c}
\hline Sample & $\begin{array}{c}J_{\mathrm{C}}(77 \mathrm{~K}) \\
\mathrm{A} / \mathrm{cm}^{2}\end{array}$ & $\begin{array}{c}J_{\mathrm{C}}(4.2 \mathrm{~K}) \\
\mathrm{A} / \mathrm{cm}^{2}\end{array}$ & $\begin{array}{c}\rho(95 \mathrm{~K}) \\
\mathrm{m} \Omega \cdot \mathrm{cm}^{2}\end{array}$ & $\begin{array}{c}J_{\mathrm{CM}}(77 \mathrm{~K}) \\
\mathrm{A} / \mathrm{cm}^{2}\end{array}$ & $\begin{array}{c}J_{\mathrm{CM}}(4.2 \mathrm{~K}) \\
\mathrm{A} / \mathrm{cm}^{2}\end{array}$ & $\begin{array}{c}\langle d\rangle \\
\mu \mathrm{m}\end{array}$ & $\begin{array}{c}\text { Density, } \\
\% \text { theor. }\end{array}$ \\
\hline YBCO-1 & 150 & 1500 & 0.45 & $2 \cdot 10^{5}$ & $5.5 \cdot 10^{6}$ & 10 & 93 \\
YBCO -2 & 15 & 170 & 2.2 & $5.6 \cdot 10^{5}$ & $7.4 \cdot 10^{6}$ & 5 & 86 \\
YBCO-3 & 5 & 100 & 6 & $1.5 \cdot 10^{5}$ & $2.3 \cdot 10^{6}$ & 4 & 88 \\
YBCO-comp. & $<0.01$ & 0.1 & 800 & $3 \cdot 10^{5}$ & $8 \cdot 10^{6}$ & 2 & $\sim 60$
\end{tabular}

\section{3. Результаты и обсуждение}

\section{1. Внутригранульный, межгранульный критический ток и резистивный переход}

По данным магнитных измерений температура появления диамагнитного сигнала из магнитных измерений составили $93,92.5,90.5$ и $93.5 \mathrm{~K}$ для образцов ҮВСО-1, YВСО-3, YВСО-3 и YВСО-сотр соответственно. При этих же температурах наблюдается начало резистивного перехода (резкое уменьшения сопротивления). Таблица содержит данные по плотности критического тока $J_{\mathrm{C}}$ исследованных образцов. Образец ҮВСО-1 обладает весьма высоким значением $J_{\mathrm{C}}$, как при гелиевой, так и при азотной температурах. При синтезе образцов YВСО-2 и YВСО-3 специальных мер для достижения больших значений $J_{\mathrm{C}}$ не предпринималось, и видно, что критический ток этих образцов значительно (на порядок) меньше. При этом увеличиваются и значения удельного электросопротивления $\rho$ в нормальном $(95 \mathrm{~K})$ состоянии, см. таблицу, а также есть корреляция со значением физической плотности. В композитных ВТСП, приготовленных методом быстрого спекания, несверхпроводящий ингредиент выступает в роли межгранульных границ [72,53], и введение диэлектрика $[72,53,14]$ приводит к дополнительному ослаблению джозефсоновской связи и, как следствие, к очень низким величинам $J_{\mathrm{C}}$, а также большому удельному сопротивлению в нормальном состоянии.

На рис. 2 приведены петли магнитного гистерезиса исследованных образцов при температурах $77 \mathrm{~K}(a)$ и $4.2 \mathrm{~K}(b)$. При $T=4.2 \mathrm{~K}$ зависимости $M(H)$ почти симметричны относительно оси абсцисс, а при $77 \mathrm{~K}$ такого уже не наблюдается (см. левую вставку рис. 2, a). Появление асимметрии при высоких температурах связано с ослаблением пиннинга абрикосовских вихрей в приповерхностном слое гранул $[73,74]$. По абсолютной величине намагниченность (при определенном поле) несколько различается для исследованных образцов. Из значений намагниченности полной петли гистерезиса можно получить величину плотности внутригранульного критического тока $J_{\mathrm{CM}}$ [75]. Согласно выражению, следующему из модели Бина,

$$
J_{\mathrm{CG}}\left(\mathrm{A} / \mathrm{cm}^{2}\right)=30 \Delta M\left(\mathrm{emu} / \mathrm{cm}^{3}\right) / d(\mathrm{~cm}),
$$

где $d-$ средний размер гранул, $\Delta M=M\left(H_{\text {inc }}\right)$ $-M\left(H_{\mathrm{dec}}\right)$ при $H_{\mathrm{inc}}=H_{\mathrm{dec}}$. Значения $J_{\mathrm{CM}}$ во внешнем поле $H \sim 100$ Ое, полученные по выражению (3), приведены в таблице. Видно, что значения $J_{\mathrm{CM}}$ для серии
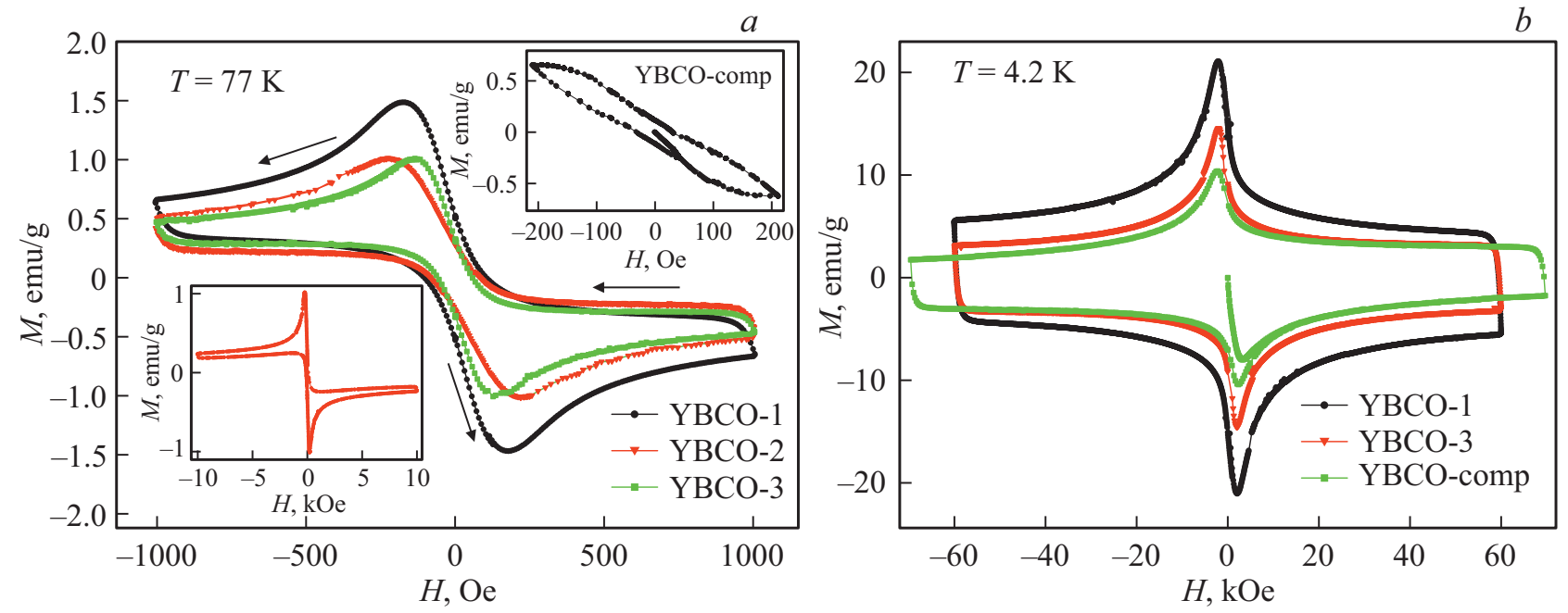

Рис. 2. Петли магнитного гистерезиса $M(H)$ исследованных образцов при $T=77 \mathrm{~K}(a)$ и $4.2 \mathrm{~K}(b)$. Левая вставка на $(a)-$ $M(H)$ образца YВСО-2 до максимально приложенного поля $\left|H_{\max }\right|=10 \mathrm{kOe.} \mathrm{Правая} \mathrm{вставка} \mathrm{на}(a)-M(H)$ образца ҮВСО-сотр. Стрелки показывают направление изменения (возрастания или убывания) внешнего поля $H$. 

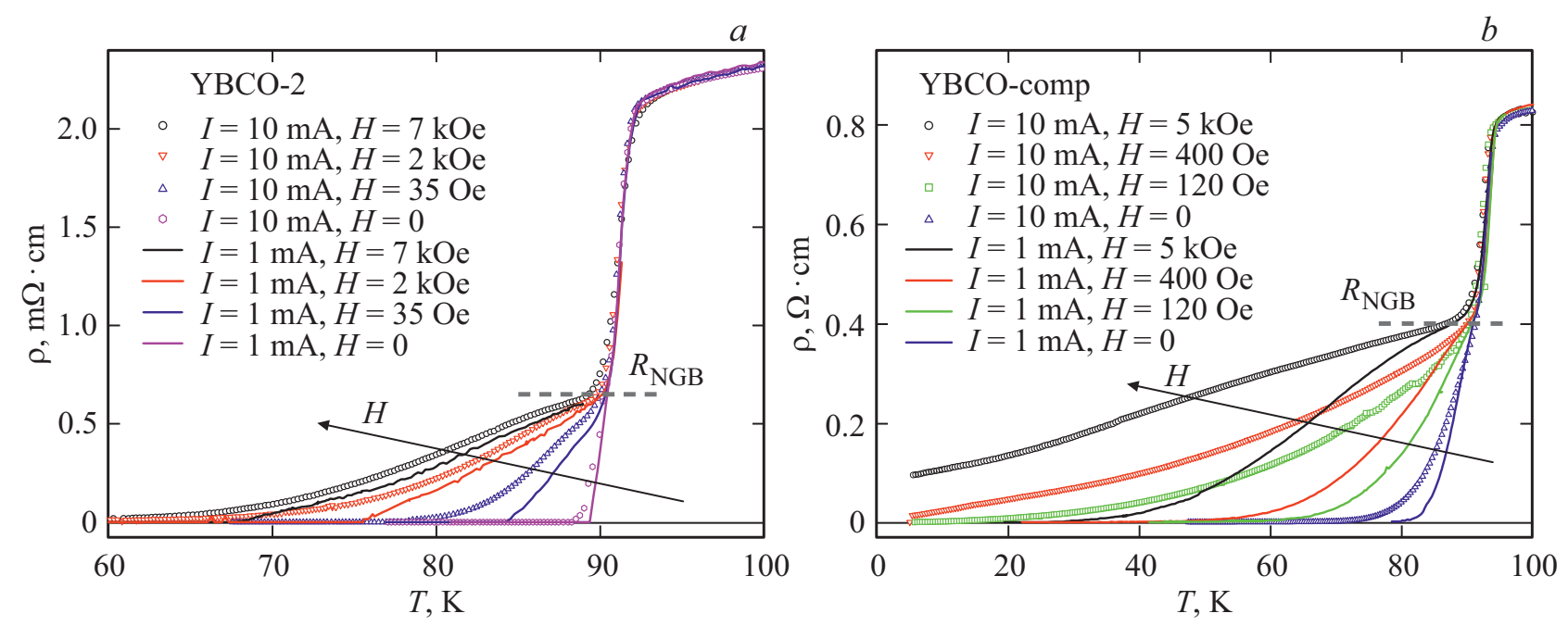

Рис. 3. Зависимости $R(T)$ для YВСО-2 (a) и YВСО-comp $(b)$ при указанных значениях внешнего поля $H$ транспортного тока $I$ (плотности тока при одном и том же значении $I$ примерно одинаковы для обоих образцов). Указано „нормальное“ сопротивление подсистемы межгранульных границ $-R_{\mathrm{NGB}}$.

исследованных образцов - величины одного порядка. Отметим, что сравнивая данные по транспортному $J_{\mathrm{C}}$ и „магнитному“ (внутригранульному) $J_{\text {См критическому }}$ току можно убедиться в справедливости строгого неравенства $J_{\mathrm{CM}} \gg J_{\mathrm{C}}$, следующего из концепции двухуровневой сверхпроводящей системы в гранулярных ВТСП.

Разница в значениях плотности транспортного критического тока $J_{\mathrm{C}}$ (таблица) исследованных образцов, а фактически - в энергии джозефсоновской связи, проявляется и в степени уширения сверхпроводящего перехода под действием внешнего магнитного поля и транспортного тока. Рис. 3 иллюстрирует зависимости $R(T)$ для выборочных образцов - YВСО-2 (рис. 3, a) и ҮВСО-сотр (рис. 3, $b$ ) в различных внешних полях и двух значениях тока. Двухступенчатый вид зависимостей $R(T)$, видимый на рис. 3, отражает наличие двух сверхпроводящих подсистем [2,12-14,21-23,47,60,52-54,62,76-78]; резкий скачок сопротивления соответствует сверхпроводящему переходу в ВТСП гранулах, а затянутый „хвост“ $R(T)$ отражает процессы диссипации в подсистеме межгранульных границ. Величина плавной части зависимостей $R(T)$ для разных значений $H$ и $I$ в окрестности начала их расхождения (после перехода в гранулах) соответствует полному или „нормальному“ сопротивлению (аналог $R_{\mathrm{N}}$ в выражении (2)) подсистемы межгранульных границ, обозначаемому далее $R_{\mathrm{NGB}}$. Диапазон магнитных полей для данных рис. 3 (до 5-8 kOе) достаточно мал чтобы привести к заметному уширению сверхпроводящего перехода в гранулах, однако в подсистеме межгранульных границ уширение резистивного перехода до некоторой

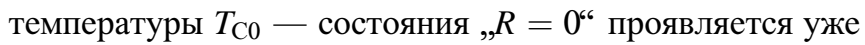
в достаточно слабых внешних полях. Также из рис. 3 видно, что и десятикратное увеличение транспортного тока также уширяет резистивный переход. При сравне-

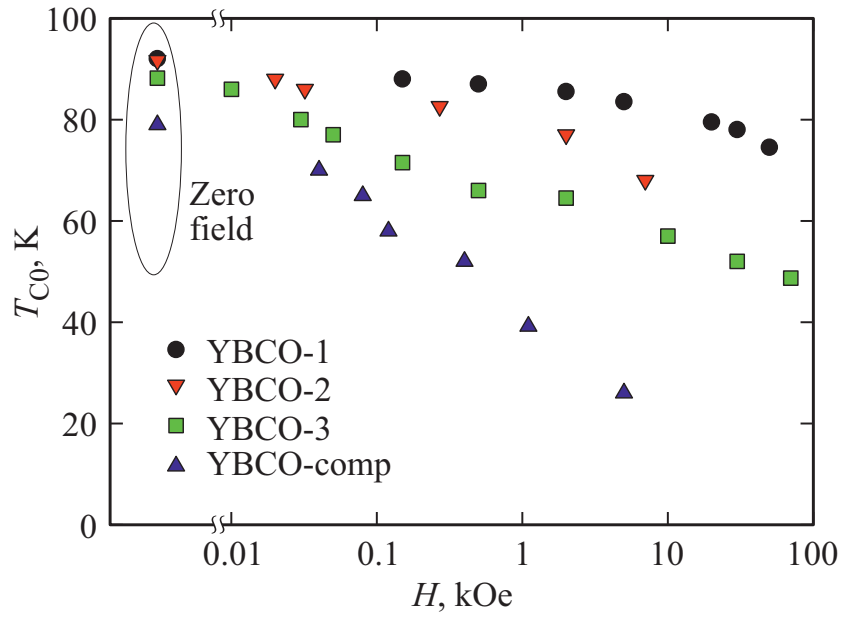

Рис. 4. Зависимости температуры $T_{\mathrm{C} 0}$ (состояния „R $R=0^{\star}$, см. рис. 3) от внешнего поля $H$ для серии исследованных образцов. Ось $H-$ логарифмическая шкала, точка с „,H=0“ соответствует $0.5 \mathrm{Oe}$.

нии степени уширения резистивного перехода образцов YBCO-2 и YВCO-comp (значения внешнего поля тока примерно одинаковы для рис. $3, a$ и рис. $3, b)$ видно, как в композите, благодаря внедрению $\mathrm{CuO}$, значительно ухудшены межгранульные границы.

Рис. 4 иллюстрирует зависимости величин температуры $T_{\mathrm{C} 0}$ для исследованных образцов от внешнего поля (логарифмическая шкала). Отметим, что эти данные получены при примерно одинаковых плотностях транспортного тока (транспортный ток $I=1 \mathrm{~mA}$ ), и, учитывая близость температур начала сверхпроводящего перехода для всех образцов, можно считать, что степень уменьшения величин $T_{\mathrm{C} 0}$ с ростом поля обратно пропорциональна энергии джозефсоновской связи. Ана- 

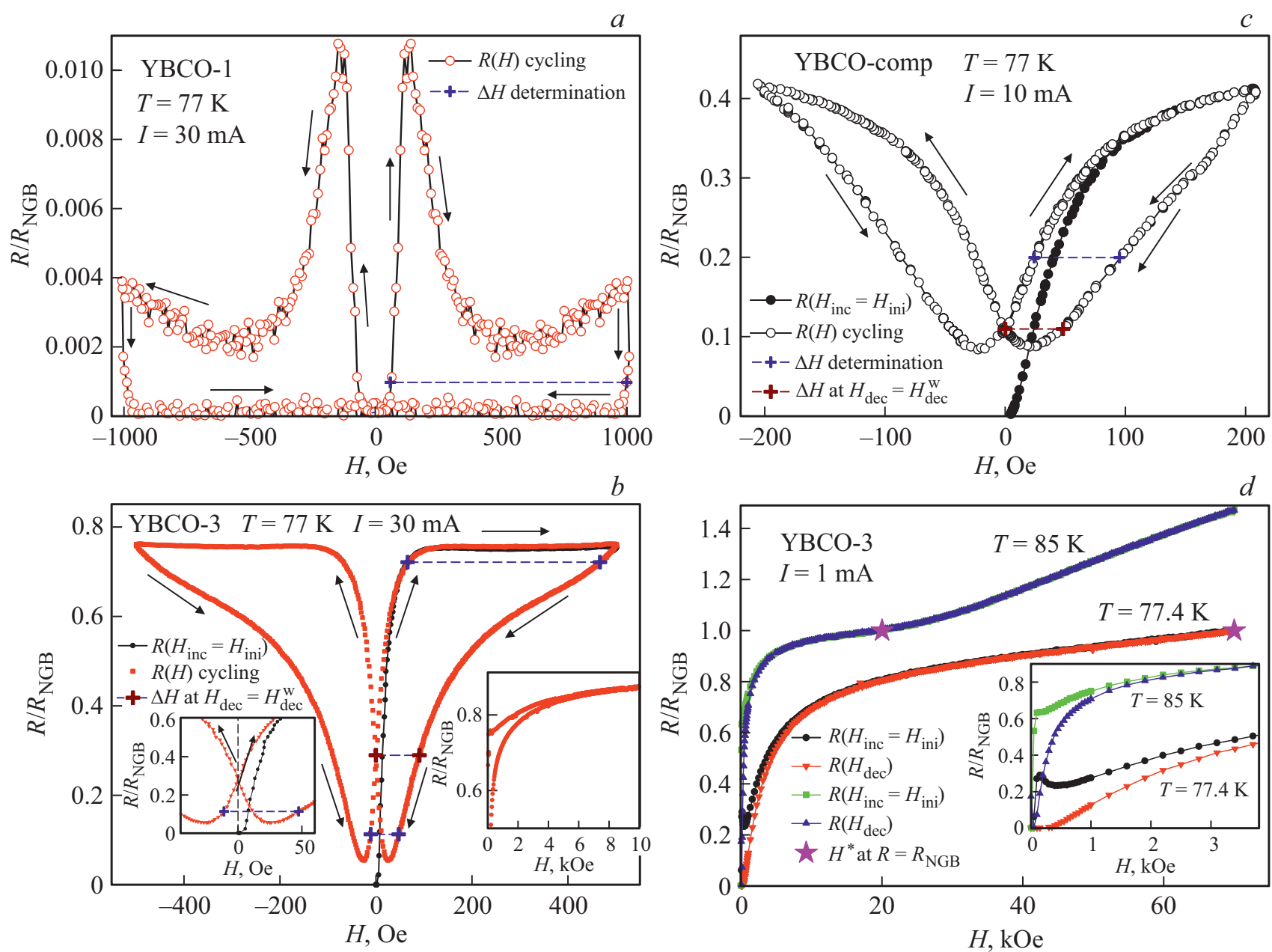

Рис. 5. Типичные гистерезисные зависимости магнитосопротивления исследованных образцов при относительно высоких температурах. Данные по сопротивлению нормированы на величину $R_{\mathrm{NGB}}$, см. рис. 3. Стрелки показывают направление изменения внешнего поля $H$. Показаны как начальные ветки $-R\left(H_{\mathrm{inc}}=H_{\mathrm{ini}}\right)(b, c, d)$, так и зависимости $R\left(H_{\mathrm{inc}}\right)$ и $R\left(H_{\mathrm{dec}}\right)$ при циклировании внешнего поля до одинаковой величины $\pm H_{\max }(a, b, c)$. Зависимости $R(H)$ на $(d)$ содержат начальные ветки $R\left(H_{\text {inc }}=H_{\text {ini }}\right)$ и обратный ход $R\left(H_{\text {dec }}\right)$ при указанных температурах; также указано поле $H^{*}$, при котором начинается диссипация в гранулах $\left(R\left(H^{*}\right) \approx R_{\mathrm{NGB}}\right)$. Штриховые горизонтальные линии на $(a, b, c)$ поясняют определение полевой ширины гистерезиса магнитосопротивления $\Delta H=H_{\mathrm{dec}}-H_{\mathrm{inc}}$ при условии $R\left(H_{\mathrm{dec}}\right)=R\left(H_{\mathrm{inc}}\right)$.

лизируя данные по $J_{\mathrm{C}}$ (таблица) и зависимости $T_{\mathrm{C} 0}(H)$ (рис. 4), можно заключить, что для серии образцов YBCO-1, YBCO-2, YBCO-3, YBCO-comp наблюдается постепенное ухудшение межгранульных границ (в смысле прозрачности для носителей сверхпроводящего тока). В то же время, магнитные свойства, внутригранульный критический ток и температура перехода ВТСП гранул примерно одинаковы.

\section{2. Гистерезис магнитосопротивления и его описание}

На рис. 5 приведены типичные гистерезисные зависимости магнитосопротивления $R(H)$ при достаточно высоких температурах (77 K и выше). Зависимости $R(H)$ нормированы на значение $R_{\mathrm{NGB}}$ (см. рис. 3). Такое представление магнитосопротивления позволяет оце- нить степень резистивного отклика от межгранульных границ относительно максимально возможной величины (при $\left.R=R_{\mathrm{NGB}}\right)$. Для образцов $\mathrm{YBCO}-1$ и YВСО-3 (рис. 5, $a$ и $b$ соответственно) есть большое различие величин $R / R_{\mathrm{NGB}}$ при примерно одинаковых значениях транспортного тока $I$. Причиной этого является разный критический ток этих образцов (таблица).

Данные рис. 5 содержат практически все многообразие наблюдаемых типов зависимостей $R(H)$ гранулярных ВТСП иттриевой системы в области высоких температур. Характер гистерезисных зависимостей $R(H)$ был описан во Введении. Для данных рис. 5 в большом диапазоне полей выполняется неравенство $R\left(H_{\mathrm{inc}}\right)>R\left(H_{\mathrm{dec}}\right)$. В то же время для начальной ветки магнитосопротивления $R\left(H_{\text {ini }}=H_{\text {inc }}\right)$ есть участок в области полей вблизи $H=0$ в котором имеет место обратная ситуация: $R\left(H_{\text {ini }}\right)<R\left(H_{\mathrm{dec}}\right)$, см. левую вставку 
рис. $5, b$ и рис. $5, c$. Немонотонное поведение зависимости $R\left(H_{\mathrm{inc}}\right)$ проявляется в виде яркой особенности для образца ҮВСО-1 (рис. 5,a). Для образца YВСО-3 локальный максимум выражен в гораздо меньшей степени (рис. 5, $b$ ). Согласно результатам работ [39,65-70], наличие локального максимума зависимости $R\left(H_{\text {inc }}\right)$, соответствует минимуму на зависимости $M\left(H_{\text {inc }}\right)$, ввиду того, что зависимость $M(H)$ входит в выражение (1) для эффективного поля, а магнитосопротивление является функцией $B_{\text {eff }}$ - выражение (2). Минимум на обратном ходу магнитосопротивления, видимый на рис. $5, b$ и левой вставке к нему (образец ҮВСО-3), а также рис. 5, $c$ (образец ҮВСО-сотр), соответствует ситуации, когда внешнее поле в наибольшей степени компенсируется полем $B_{\text {ind }}$, индуцированном гранулами, см. рис. $1, b$. Для образца ҮВСО-1, при условиях эксперимента рис. 5, $a$, относительно небольшой измерительный ток меньше критического тока при величине эффективного поля $B_{\text {eff }}\left(H_{\text {dec }}\right)$ для ветки спадающего поля, поэтому на обратном ходу магнитосопротивление $R\left(H_{\mathrm{dec}}\right)=0$. Зависимости $R(H)$ на рис. 5, $a, b, c$ были получены при циклировании для максимальных полей $H_{\max }$ не более $\pm 1 \mathrm{kOe;} \mathrm{в} \mathrm{бо́льших} \mathrm{полях} \mathrm{зависимость} R(H)$ плавно приближается к значению $R_{\mathrm{NGB}}$, что показано на правых вставках рис. $5, b$ и рис. $5, d$, и при этом в достаточно больших полях гистерезис становится достаточно узким.

Выполнение строгого неравенства $J_{\mathrm{CM}} \gg J_{\mathrm{C}}$ означает отсутствие диссипации в гранулах. Начало диссипации в гранулах сопровождается дополнительным резким ростом магнитосопротивления в поле $H^{*}$, соответствующем нулевому внутригранульному критическому току $J_{\mathrm{CM}}\left(H^{*}\right)=0$, или условию $\Delta M\left(H^{*}\right)=0[76,40,47,77]$. На рис. 5, $d$ показаны зависимости $R(H)$ образца ҮВСО-3 при $T=77.4$ и $85 \mathrm{~K}$. Видно, что достаточно резкая смена зависимости $R(H)$ при $T=85 \mathrm{~K}$ происходит при значении сопротивления $R \approx R_{\mathrm{NGB}}$ (при этом величина $R_{\mathrm{NGB}}$ была получена независимо, из зависимостей $R(T)$ в различных полях). Для $T=85 \mathrm{~K}$ величина $H^{*} \approx 20 \mathrm{kOe}$, и при понижении температуры характерное поле $H^{*}$ увеличивается (при $T=77.4 \mathrm{~K} H^{*}$ составляет около $70 \mathrm{kOe})$. Отметим, что плотности транспортного тока, которые возможно варьировать в экспериментах, значительно меньше $J_{\mathrm{CM}}$, поэтому наблюдать уменьшение $H^{*}$ из магнитотранспортных измерений, по крайней мере, для гранулярных ВТСП иттриевой системы, практически невозможно.

Объяснение гистерезисного поведения $R(H)$ основано в рамках концепции рассмотрения эффективного поля в межгранульной среде, определяемого выражением (1). Упрощая выражение (2), магнитосопротивление пропорционально эффективному полю: $R(H) \sim B_{\text {eff }}(H)$. Покажем на примере выборочных данных пример качественного описания наблюдаемой формы гистерезиса $R(H)$. Рис. 6, $a$ иллюстрирует зависимости $B_{\text {eff }}(H)$, полученные по выражению (1) с использованием экспериментальных данных $M(H)$ для образца YBCO-1 (рис. 2,a) и различных значений $\alpha$. Прежде всего, стоит отметить
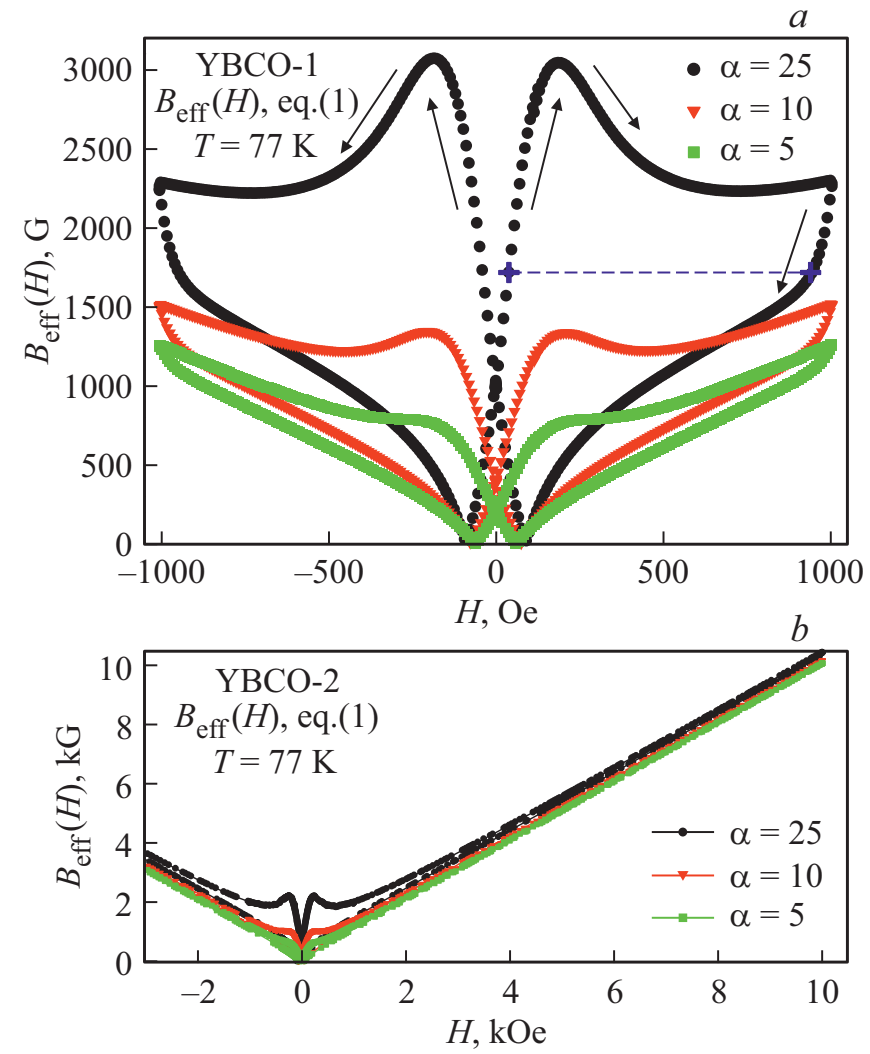

Рис. 6. Гистерезисные зависимости эффективного поля $B_{\mathrm{eff}}(H)$ при $T=77 \mathrm{~K}$, построенные по выражению (2) при различных значениях параметра $\alpha$ (указаны на рисунке) с использованием данных по намагниченности (рис. 2) образцов YBCO-1 (a) при $\left|H_{\max }\right|=1 \mathrm{kOе}$ и YВСО-2 (b) при $\left|H_{\max }\right|=10 \mathrm{kOе.} \mathrm{Стрелки}$ показывают направление изменения внешнего поля $H$. Штриховая горизонтальная линия на $(a)$ поясняет определение полевой ширины гистерезиса эффективного поля $\Delta H=H_{\mathrm{dec}}-H_{\mathrm{inc}}$ при условии $B_{\text {eff }}\left(H_{\text {dec }}\right)=B_{\text {eff }}\left(H_{\text {inc }}\right)$.

сходство формы гистерезиса зависимостей $B_{\text {eff }}(H)$ и $R(H)$ : имеет место неравенство $B_{\text {eff }}\left(H_{\text {inc }}\right)>B_{\text {eff }}\left(H_{\text {dec }}\right)$. Ясно, что экспериментальный гистерезис намагниченности входит в выражение (1) в ,перевернутом“ виде, и его относительный вклад в $B_{\text {eff }}$ определяется величиной $\alpha$. Из данных рис. $6, a$ видно, что при значении $\alpha$ около 10 и больше, отчетливо проявляется максимум зависимости $B_{\text {eff }}\left(H_{\text {inc }}\right)$, соответствующий максимуму зависимости $R\left(H_{\text {inc }}\right)$. Минимум зависимости $B_{\text {eff }}\left(H_{\mathrm{dec}}\right)$ также воспроизводится в рамках этого подхода. Сам эффект сгущения потока выражается в значительно большей величине

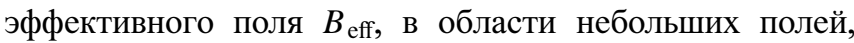
чем само значение внешнего поля $H$, см. рис. $6, b$. В больших полях в выражении (1) вклад, связанный с намагниченностью, уже незначительный, и $B_{\mathrm{eff}} \approx H$, см. рис. $6, b$.

Зависимость $R\left(B_{\text {eff }}\right)$ в рамках описываемого подхода уже безгистерезисна: $\quad R\left(B_{\text {eff }}\left(H_{\text {inc }}\right)\right)=R\left(B_{\text {eff }}\left(H_{\mathrm{dec}}\right)\right)$ при $\quad B_{\text {eff }}\left(H_{\text {inc }}\right)=B_{\text {eff }}\left(H_{\text {dec }}\right) . \quad$ Следовательно, можно оперировать с такой характеристикой зависимостей 
$R(H) \quad$ и $B_{\text {eff }}(H)$, как полевая ширина гистерезиса: $\Delta H=H_{\mathrm{dec}}-H_{\text {inc }}$ при условии $R\left(H_{\mathrm{inc}}\right)=R\left(H_{\mathrm{dec}}\right)$, либо $B_{\text {eff }}\left(H_{\text {inc }}\right)=B_{\text {eff }}\left(H_{\text {dec }}\right)$. Ранее было показано, что для гистерезисных зависимостей $R(H)$ гранулярных ВТСП величина $\Delta H$ не зависит от величины транспортного тока в достаточно широких пределах варьирования тока $[63,64,61,47,67,69]$. Следовательно, даже для качественного описания гистерезиса $R(H)$ необходимо согласие между величиной $\Delta H$, полученной из экспериментальных зависимостей $R(H)$ и $\Delta H$, воспроизводимой зависимостью $B_{\text {eff }}(H)$. Гистерезис $R(H)$ в полях до $H_{\max } \sim \pm 1 \mathrm{kOе}$ достаточно широкий, см. рис. $5, a, b$, и для воспроизведения схожих величин $\Delta H$ зависимости $B_{\text {eff }}(H)$ необходимо, чтобы значение параметра $\alpha$ находилось в диапазоне 20-25. Что касается данных в большем диапазоне полей, то здесь гистерезис $R(H)$ становится узким (см. правую вставку рис. $5, b$ и $d$ ); в этом диапазоне полей гистерезис намагниченности достаточно мал (см. левую вставку рис. 2,b), и, как следствие, гистерезис $B_{\text {eff }}(H)$ также узкий, см. рис. $6, b$. Отметим, что для лучшего и детального описания гистерезиса $R(H)$, необходимо рассматривать зависимость параметра $\alpha$ от внешнего поля.

В низкой температуре $(4.2 \mathrm{~K})$ характер гистерезиса $R(H)$ остается тем же: $R\left(H_{\text {inc }}\right)>R\left(H_{\mathrm{dec}}\right)$, см. рис. 7, на котором приведены зависимости $R(H)$ образцов YBCO-1 и YВСО-3. Для измерения магнитосопротивления при низких температурах уже необходимы значительно бо́льшие плотности транспортного тока, чем при высоких температурах. При этом увеличивается и интервал внешнего поля, в котором гистерезис $R(H)$ широкий. Это соответствует объяснению в рамках выражения (1)

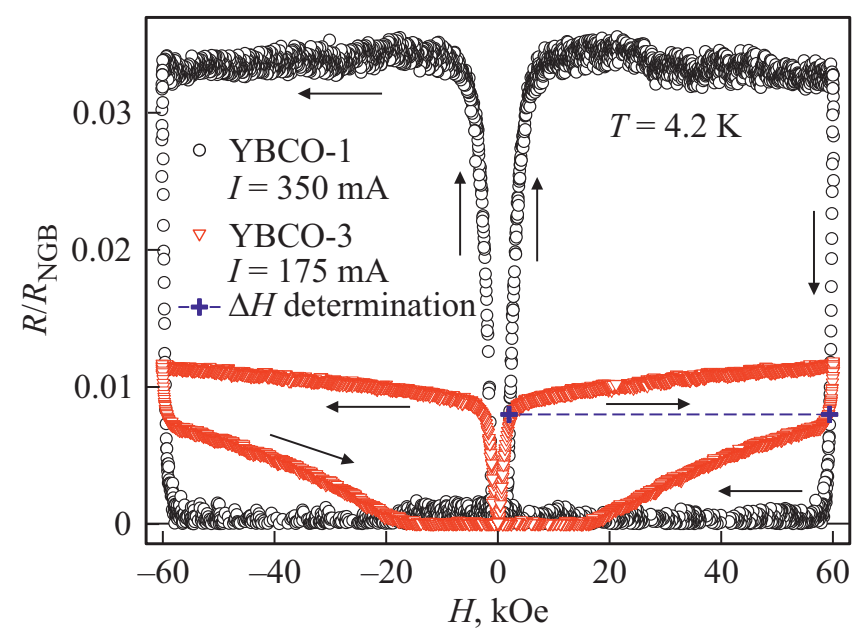

Рис. 7. Гистерезисные зависимости $R(H)$ образцов ҮВСО-1 и ҮВСО-3 при $T=4.2 \mathrm{~K}$, полученные при циклировании внешнего поля до $\pm 60 \mathrm{kOe}$. Данные по сопротивлению нормированы на величину $R_{\mathrm{NGB}}$, см. рис. 3. Стрелки показывают направление изменения внешнего поля $H$. Штриховая горизонтальная линия поясняют определение полевой ширины гистерезиса магнитосопротивления $\Delta H=H_{\mathrm{dec}}-H_{\mathrm{inc}}$ при условии $R\left(H_{\mathrm{dec}}\right)=R\left(H_{\mathrm{inc}}\right)$.
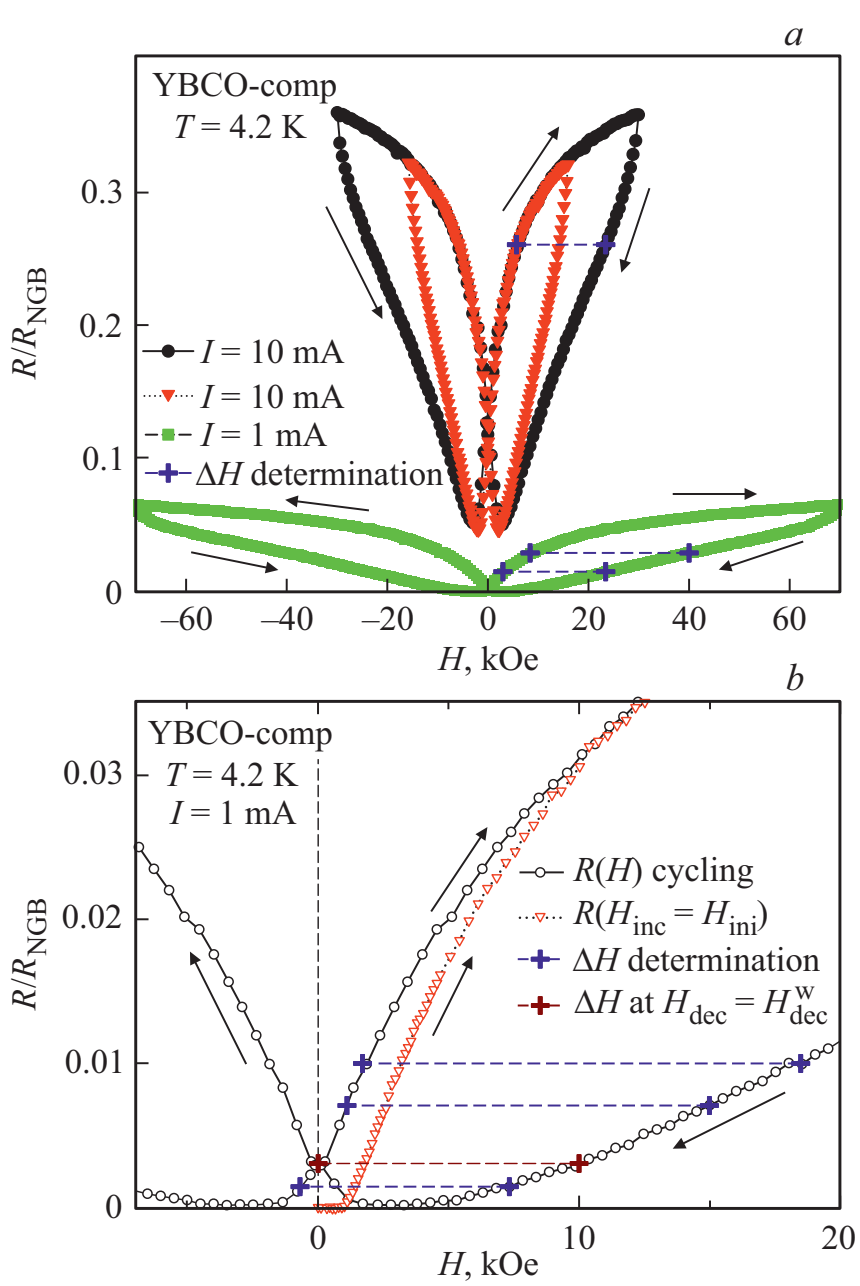

Рис. 8. Гистерезисные зависимости $R(H)$ образца ҮВСО-сотр при $T=4.2 \mathrm{~K}$, полученные при различных величинах транспортного тока (указаны на рисунках) и циклирования поля до различных значений $H_{\max }$. На $(b)$ показано относительное расположение начальной ветки магнитосопротивления $R\left(H_{\mathrm{inc}}\right)$ и $R(H)$ при циклировании внешнего поля. Данные по сопротивлению нормированы на величину $R_{\mathrm{NGB}}$, см. рис. 3 . Стрелки показывают направление изменения внешнего поля $H$. Штриховые горизонтальные линии поясняют определение полевой ширины гистерезиса магнитосопротивления $\Delta H=H_{\mathrm{dec}}-H_{\mathrm{inc}}$ при условии $R\left(H_{\mathrm{dec}}\right)=R\left(H_{\mathrm{inc}}\right)$.

с учетом, того, что и абсолютные значения намагниченности увеличились более чем на порядок (рис. 2, $b$ ), значит, и эффективное поле также сильно различается для ветвей прямого и обратного хода. Обращает на себя отсутствие выраженного максимума зависимостей $R\left(H_{\mathrm{inc}}\right)$, хотя, в отличие от данных рис. $5, b$, величины $R / R_{\mathrm{NGB}}$ далеки от своей максимальной величины ${ }^{1}$. Возможные причины отсутствия выраженного максимума, который, соответствует минимуму зависимости $M(H)$ (рис. $2, b)$,

\footnotetext{
${ }^{1}$ Если, межгранульные границы обладают металлическим характером проводимости $[3,6]$, можно ожидать уменьшение $R_{\mathrm{NGB}}$ с понижением температуры, что приведет к несколько большей величине $R_{\mathrm{NGB}}$ для данных рис. 7 .
} 
и, согласно выражению (1), ожидается в районе $\sim 2 \mathrm{kOe}$, обсуждалось в работе [69]. Для композитного образца вид гистерезиса $R(H)$ и относительное расположение начальной ветки магнитосопротивления $R\left(H_{\mathrm{ini}}=H_{\mathrm{inc}}\right)$ и зависимостей $R\left(H_{\mathrm{inc}}\right), R\left(H_{\mathrm{dec}}\right)$ при низких температурах, см. рис. 8, остается таким же, как и для данных при высоких температурах.

\section{3. Универсальное поведение ширины гистерезиса $\boldsymbol{R}(\boldsymbol{H})$}

Проанализируем поведение ширины гистерезиca магнитосопротивления $\quad \Delta H=H_{\mathrm{dec}}-H_{\mathrm{inc}} \quad$ (при $\left.R\left(H_{\mathrm{dec}}\right)=R\left(H_{\mathrm{inc}}\right)\right)$ для различных образцов при различных температурах. Будем рассматривать полную петлю гистерезиса $R(H)$, получаемую при циклировании внешнего поля (без начального хода $\left.R\left(H_{\text {ini }}=H_{\text {inc }}\right)\right)$. Фактически, ширина гистерезиса $R(H)$ - это длина горизонтального отрезка между точками спадающей $R\left(H_{\mathrm{dec}}\right)$ и возрастающей $R\left(H_{\mathrm{inc}}\right)$ ветвями гистерезиса магнитосопротивления. Типичные примеры определения $\Delta H$ показаны на рис. $5, a, b, c$, рис. 7 , рис. $8, a$ (штриховые горизонтальные линии). Значение $\Delta H$, должно всегда быть меньше $H_{\mathrm{dec}}$, пока в некотором поле $H_{\mathrm{dec}}^{\mathrm{w}}$ величина сопротивления $R\left(H_{\mathrm{dec}}=H_{\mathrm{dec}}^{\mathrm{w}}\right)$ не будет равной $R\left(H_{\mathrm{inc}}=0\right)$. Тогда однозначно, $\Delta H\left(H_{\mathrm{dec}}=H_{\mathrm{dec}}^{\mathrm{w}}\right)=H_{\mathrm{dec}}$, cм. pис. 5, $b, c$ и $8, b$. В диапазоне $0 \leq H<H_{\mathrm{dec}}^{\mathrm{w}} \Delta H$ уже будет больше значения $H_{\mathrm{dec}}$. Значение поля $H_{\mathrm{dec}}^{\mathrm{w}}$ не превышает 50-60 Ое для данных при высоких температурах (рис. $5, b, c)$ и $10 \mathrm{kOe}$ при $4.2 \mathrm{~K}$ для образца YВСО-comp (рис. $8, b$ ), что значительно меньше максимального поля Нmax. Отметим, что если в некотором диапазоне $H<H_{\text {dec }}$ сопротивление равно нулю (см. рис. 5, $a$ и рис. 7), то здесь величина $\Delta H$ не определена.

На рис. 9 приведены зависимости $\Delta H\left(H_{\mathrm{dec}}\right)$, полученные из экспериментальных данных $R(H)$ при различных температурах для образцов ҮВСО-1, YВСО-2 и YВСО-3. На этом рисунке использована двойная логарифмическая шкала ввиду различного диапазона максимального приложенного поля для низкой температуры (10-60 kOe) и высоких температур (1-10 kOe). Для данных при высоких температурах в диапазоне полей $H_{\mathrm{dec}}$ более $\sim 1 \mathrm{kOe}$, как и говорилось при обсуждении гистерезиса $R(H)$, см. п.п. 3.2., значения $\Delta H$ малы (см. данные для образца ҮВСО-2 при $T=77 \mathrm{~K}$ ), а диапазоне полей $0 \leq H<H_{\mathrm{dec}}^{\mathrm{m}}$ выполняется неравенство $\Delta H\left(H_{\mathrm{dec}}=H_{\mathrm{dec}}^{\mathrm{w}}\right)>H_{\mathrm{dec}}$. Кроме того, для образца YBCO-1 магнитосопротивление на обратном ходу, практически скачкообразно (рис. 7), уменьшается до нуля, и зависимость $\Delta H\left(H_{\mathrm{dec}}\right)$ содержит, буквально, одну точку, приведенную на рис. 9.

Основное, что обращает на себя внимание на рис. 9, это близость полученных экспериментальных данных $\Delta H\left(H_{\mathrm{dec}}\right)$ линейной функции $\Delta H=H$ (показана на рис. 9, $a$ сплошной линией) в большом диапазоне поля $H_{\mathrm{dec}}$, и идентичность характера зависимостей $\Delta H\left(H_{\mathrm{dec}}\right)$ для различных образцов при различных температурах.

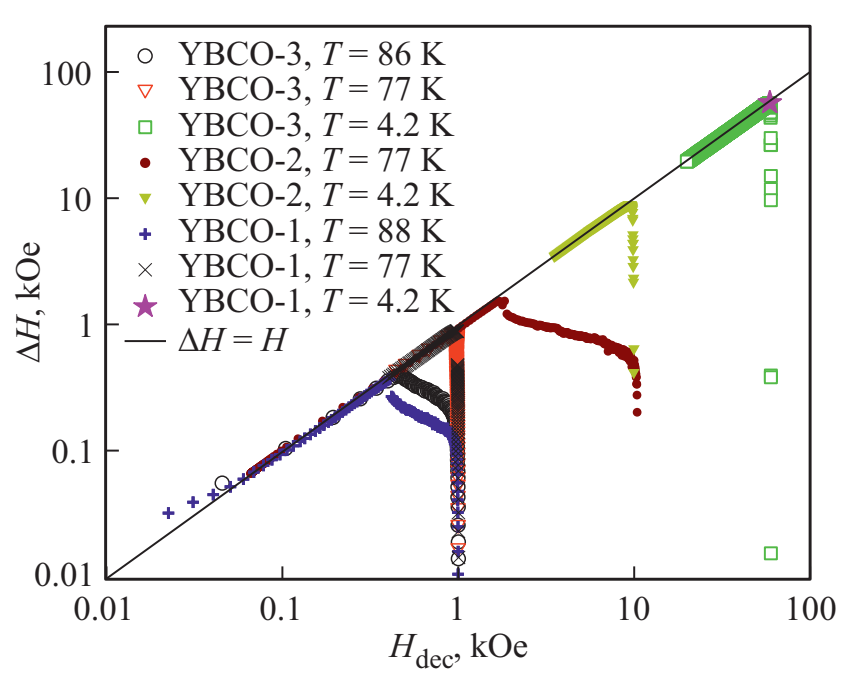

Рис. 9. Полевая ширина гистерезиса магнитосопротивления $\Delta H$ от поля $H_{\mathrm{dec}}$ (использована двойная логарифмическая шкала) для образцов YВСО-1, YВСО-2, YВСО-3 (символы) при указанных температурах и различных значениях $H_{\max }$. Сплошная линия - линейная зависимость $\Delta H=H_{\mathrm{dec}}$.

Следование зависимости $\Delta H \approx H_{\mathrm{dec}}$ означает, что зависимость $R\left(H_{\text {inc }}\right)$ достаточно быстро возрастает, и по достижении некоторого поля $H_{\mathrm{inc}}^{\mathrm{m}}$ выходит на слабую зависимость от поля, тогда, если $H_{\text {inc }}^{\mathrm{m}}$ достаточно мала, по сравнению с $H_{\max }$, логично, что $\Delta H \approx H_{\text {dec. }}$ Весьма неожиданным оказалось, что, несмотря на большие различия в транспортном критическом токе (таблица), магнитотранспортных свойствах (рис. 3), а также форме зависимостей $R(H)$ (рис. 5, 7), все исследованные образцы иттриевого ВТСП проявляют некое универсальное поведение гистерезиса магнитосопротивления при различных температурах.

Ширина гистерезиса $R(H)$ должна совпадать с шириной гистерезиса эффективного поля $B_{\text {eff }}(H)$. Подставляя поочередно в (1) $H=H_{\text {inc }}$ и $H=H_{\text {dec }}$, и вычитая полученные выражения одно из другого, получим при $B_{\text {eff }}\left(H_{\text {dec }}\right)=B_{\text {eff }}\left(H_{\text {inc }}\right)$ :

$$
\Delta H=4 \pi \alpha\left\{M\left(H_{\mathrm{dec}}\right)-M\left(H_{\mathrm{inc}}\right)\right\} .
$$

Как и говорилось ранее, здесь принято, что параметр $\alpha$ не зависит от внешнего поля. Тогда идентичность поведения $\Delta H$ от $H_{\text {dec }}$ для различных температур и различных образцов может объясняться примерно одинаковым поведением гистерезиса намагниченности, а также его температурной эволюцией. С другой стороны, параметр $\alpha$ достаточно большой, и, видимо, практически не зависит от температуры [67-69,79]. Тогда обнаруженное универсальное поведение ширины гистерезиса магнитосопротивления, проиллюстрированное на рис. 9, может быть качественно объяснено. Фактически, параметр $\alpha$ определяет эффективное сгущение линий магнитной индукции от магнитных моментов гранул в области межгранульной границы. Известно, что протяженность межгранульных границ в ВТСП иттриевой системы крайне 


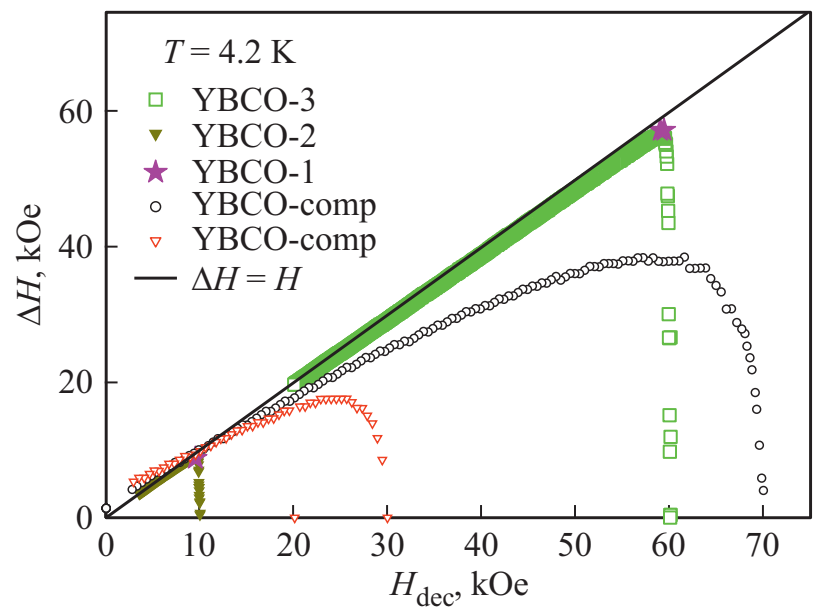

Рис. 10. Полевая ширина гистерезиса магнитосопротивления $\Delta H$ от поля $H_{\mathrm{dec}}$ для исследованных образцов (символы) при $T=4.2 \mathrm{~K}$ (значения $H_{\max }$ различны). Сплошная линия линейная зависимость $\Delta H=H_{\text {dec. }}$.

мала, и, логично, что бо́льшие значения $J_{\mathrm{C}}$ соответствуют наименьшим по протяженности (толщине $l$ ) межгранульным границам [3-6]. В работе [6] были проведены оценки величины $l$ различных ВТСП образцов иттриевой системы (оценки сделаны на основании сопоставления экспериментальных и теоретических [80] температурных зависимостей критического тока при $H=0$ ). Для наилучших образцов $\left(J_{\mathrm{C}}(T=4.2 \mathrm{~K}) \sim 1.4 \mathrm{kA} / \mathrm{cm}^{2}\right)$ величина $l$, составила всего $\sim 1 / 10$ от значения длины когерентности $\xi_{0}$, тогда как для образца со значением $J_{\mathrm{C}}$ на порядок меньшим, толщина $l$ увеличилась до $\approx 1 \xi_{0}$. Для серии образцов YВСО-1,2,3 логично, что уменьшение $J_{\mathrm{C}}$ (таблица) будет соответствовать увеличению толщины межгранульных границ. При этом такое увеличение толщины межгранульных прослоек должно сказаться на величине параметра $\alpha$, однако обнаруженная идентичность поведения ширины гистерезиса $R(H)$ указывает на примерно одинаковую степень сгущения магнитного потока. Возможно, что при приложении внешнего поля есть и дополнительный эффект, заключающийся в том, что в поле (имеется виду эффективное поле) и часть приповерхностной области ВТСП гранул уже ведет себя как межгранульная прослойка. Тогда можно ожидать примерно одинаковую степень сгущения потока в межгранульной среде для исследованной серии образцов с различной токонесущей способностью.

Описанная выше особенность - идентичное поведение ширины гистерезиса $R(H)$ для различных образцов гранулярного уже не выполняется для композитного образца. На рис. 10 приведены данные по $\Delta H\left(H_{\mathrm{dec}}\right)$ как для образцов YВCO-1,2,3, так и для образца YBCO-comp при температуре $4.2 \mathrm{~K}$. Видно, что данные для композита уже нельзя, даже приближенно, описать линейной „скейлинговой“ функцией, к тому же и зависимость $\Delta H\left(H_{\mathrm{dec}}\right)$ для этого образца расположена значительно ниже, чем для образцов YВСО-1,2,3. Здесь, ввиду выражения (4), уже можно говорить о меньшем сжатии потока в области межгранульных границ, чем в „чистых“ ҮВСО. Ясно, что толщина границ между ВТСП гранулами в композитном образце значительно увеличилась ${ }^{2}$; оценки эффективной толщины, для композитов с металлооксидом $\mathrm{BaPbO}_{3}$, полученных по схожей технологии и содержанием несверхпроводящего компонента, дали величину $d$ порядка $3 \xi_{0}[6]$. Следовательно, для композита логично ожидать меньшую степень сжатия потока, или меньшее значение параметра $\alpha$, что и наблюдается из данных $\Delta H\left(H_{\mathrm{dec}}\right)$ на рис. 10.

\section{4. Заключение}

Механизм гистерезиса магнитосопротивления гранулярных ВТСП объясняется процессами диссипации в межгранульных границах, и при этом в пространстве между гранулами необходимо рассматривать не внешнее поле, а эффективное поле $B_{\text {eff. }}$ Это эффективное поле является суперпозицией внешнего поля и поля, индуцированного магнитным откликом ВТСП гранул. Следовательно, магнитный гистерезис гранулярных ВТСП определяет их магнитотранспортный гистерезис.

Основным результатом данной работы можно считать обнаруженное универсальное поведение ширины гистерезиса магнитосопротивления $\Delta H=H_{\mathrm{dec}}-H_{\mathrm{inc}}$ (при условии $\left.R\left(H_{\mathrm{dec}}\right)=R\left(H_{\mathrm{inc}}\right)\right)$ гранулярных ВТСП системы $\mathrm{Y}-\mathrm{Ba}-\mathrm{Cu}-\mathrm{O}$. В достаточно большом интервале внешних полей указанный параметр $\Delta H$ имеет функциональную зависимость, близкую к линейной, и эта зависимость одинакова как для температур, близких к критической (выше $77 \mathrm{~K}$ ), так и при низкой температуре $(T=4.2 \mathrm{~K})$. Более того, данные оказались идентичными для трех образцов, обладающих различной плотностью транспортного критического тока - от больших $\left(1.5 \mathrm{kA} / \mathrm{cm}^{2}\right.$ при $\left.4.2 \mathrm{~K}\right)$ до достаточно малых $\left(100 \mathrm{~A} / \mathrm{cm}^{2}\right.$ при $4.2 \mathrm{~K}$ ) значений $J_{\mathrm{C}}$. Причиной такого независимого от „качества“ межгранульных границ универсального поведения является эффект сжатия магнитного потока в межгранульной среде. Степень сжатия потока, по-видимому, примерно одинакова для образцов с различной токонесущей способностью. Однако исключением является ВТСП-композит, в котором критический ток еще более подавлен $\left(J_{\mathrm{C}} \sim 0.1 \mathrm{~A} / \mathrm{cm}^{2}\right.$ при $\left.4.2 \mathrm{~K}\right)$ ввиду того, что несверхпроводящий ингредиент (в данном случае - $\mathrm{CuO}$ ) выступает в роли прослоек между гранулами, и, в результате эффективная протяженность границ в композите становится значительно большей, чем протяженность естественных границ в „чистом“ $\mathrm{Y}-\mathrm{Ba}-\mathrm{Cu}-\mathrm{O}$. Для такого композитного образца эффект сжатия потока в межгранульной среде „работает“ в меньшей степени.

\footnotetext{
2 Эффективная толщина границ между ВТСП гранулами в композите зависит от концентрации несверхпроводящего компонента $[72,53,23,6]$.
} 
Таким образом, достаточно сильное сжатие потока в области межгранульных границ является основной причиной наблюдаемого широкого гистерезиса магнитосопротивления, что определяет универсальное поведение ширины этого гистерезиса для образцов ВТСП иттриевой системы.

\section{Благодарности}

Авторы благодарят Д.М. Гохфельда за обсуждение результатов. Часть измерений транспортных свойств проводились на установке PPMS-6000 Центра коллективного пользования ФИЦ КНЦ СО РАН.

\section{Конфликт интересов}

Авторы заявляют, что у них нет конфликта интересов.

\section{Список литературы}

[1] L. Ji, M.S. Rzchowski, N. Anand, M. Tinkham. Phys. Rev. B 47, 470 (1993).

[2] M. Prester. Supercond. Sci. Technol. 11, 333 (1998).

[3] M.I. Petrov, D.A. Balaev, B.P. Khrustalev, K.S. Aleksandrov. Physica C 235-240, 3043 (1994).

[4] R. Gross. Physica C 432, 105 (2005).

[5] J. Mannhart. Physica C 450, 152 (2006).

[6] М.И. Петров, Д.А. Балаев, Д.М. Гохфельд. ФТТ 49, 4, 589(2007).

[7] J.H. Durrell, N.A. Rutter. Supercond. Sci. Technol. 22, 013001 (2009).

[8] X. Obradors, T. Puig, S. Ricart, M. Coll, J. Gazquez, A. Palau, X. Granados. Supercond. Sci. Technol. 25, 123001 (2012).

[9] G. Wang, M.J. Raine, D.P. Hampshire. Supercond. Sci. Technol. 30, 104001 (2017).

[10] R.J. Joshi, R.B. Hallock, J.A. Taylor. Phys. Rev. B 55, 9107 (1997).

[11] J.W.C. De Vries, G.M. Stollman, M.A.M. Gijs. Physica C 157, 406 (1989).

[12] A.C. Wright, K. Zhang, A. Erbil. Phys. Rev. B 44, 863 (1991).

[13] C. Ganey, H. Petersen, R. Bednar. Phys. Rev. B 48, 3388 (1993).

[14] H.S. Gamchi, G.J. Russell, K.N.R. Taylor. Phys. Rev. B 50, 12950 (1994).

[15] R.J. Soulen, T.L. Francavilla, W.W. Fuller-Mora, M.M. Miller, C.H. Joshi, W.L. Carter, A.J. Rodenbush, M.D. Manlief, D. Aized. Phys. Rev. B 50, 478 (1994).

[16] D.H. Liebenberg, R.J. Soulen, T.L. Francavilla, W.W. Fuller-Mora, P.C. McIntyre, M.J. Cima. Phys. Rev. B 51, 11838 (1995).

[17] R.J. Soulen, T.L. Francavilla, A.R. Drews, L. Toth, M.S. Osofsly, W.L. Lechter, E.F. Skelton. Phys. Rev. B 51, 1393 (1995).

[18] W.M. Tieran, R. Joshi, R.B. Hallock. Phys. Rev. B 48, 3423 (1993).

[19] Y. Zhao, X.B. Zuge, J.M. Xu, L. Cao. Phys. Rev. B 49, 6985 (1994).

[20] L. Urba, C. Acha, V. Bekeris. Physica C 279, 95 (1997).

[21] H. Shakeripour and M. Akhavan. Supercond. Sci. Technol. 14,234 (2001).
[22] M.R. Mohammadizadeh, M. Akhavan. Supercond. Sci. Technol. 16, 538 (2003).

[23] Д.А. Балаев, С.И. Попков, К.А. Шайхутдинов, М.И. Петров. ФТТ 48, 5, 588 (2006).

[24] М.А. Васютин. Письма ЖТФ 39, 9 (2013).

[25] В.В. Деревянко, Т.В. Сухарева, В.А. Финкель. ФТТ 60, 3, 465 (2018).

[26] Т.В. Сухарева, В.А. Финкель. Письма ЖЭТФ 108, 4, 249 (2018).

[27] Т.В. Сухарева, В.А. Финкель. ФНТ 44, 3, 258 (2018).

[28] Т.В. Сухарева, В.А. Финкель. ФНТ 46, 5, 653 (2020).

[29] S. Shifang, Z. Yong, P. Guoqian, Y. Daoq, Z. An, C. Zuyao, Q. Yitai, K. Eiyan, Z. Qirui. Europhys. Lett. 6, 4, 359 (1988).

[30] Y.J. Quian, Z.M. Tang, K.Y. Chen, B. Zhou, J.W. Qui, B.C. Miao, Y.M. Cai. Phys. Rev. B 39, 4701 (1989).

[31] P. Mune, F.C. Fonseca, R. Muccillo, R.F. Jardim. Physica C 390, 363 (2003).

[32] Н.Д. Кузьмичев. Письма в ЖЭТФ 74, 291 (2001).

[33] Н.Д. Кузьмичев. ФТТ 43, 1934 (2001).

[34] I. Felner, E. Galstyan, B. Lorenz, D. Cao, Y.S. Wang, Y.Y. Xue, C.W. Chu. Phys. Rev. B 167, 134506 (2003).

[35] Т.В. Сухарева, В.А. Финкель. ЖЭТФ 134, 922 (2008).

[36] Т.В. Сухарева, В.А. Финкель. ФТТ 50, 6, 961 (2008).

[37] В.В. Деревянко, Т.В. Сухарева, В.А. Финкель. ЖТФ 78, 36 (2008).

[38] Т.В. Сухарева, В.А. Финкель. ФТТ 52, 3, 424 (2010).

[39] К.А. Шайхутдинов, Д.А. Балаев, С.И. Попков, М.И. Петров. ФТТ 51, 1046 (2009).

[40] D.A. Balaev, S.I. Popkov, S.V. Semenov, A.A. Bykov, K.A. Shaykhutdinov, D.M. Gokhfeld, M.I. Petrov. Physica C 470, 61 (2010).

[41] A. Altinkok, K. Kilic, M. Olutas, A. Kilic, J. Supercond. Nov. Magn. 26, 3085 (2013).

[42] Д.А. Балаев, С.И. Попков, К.А. Шайхутдинов, М.И. Петров, Д.М. Гохфельд, ФТТ 56, 8, 1492 (2014).

[43] J.E. Evetts, B.A. Glowacki. Cryogenics 28, 641 (1988).

[44] M.E. McHenry, P.P. Maley, J.O. Willis. Phys. Rev. B 40, 2666 (1989).

[45] E. Altshuler, J. Musa, J. Barroso, A.R.R. Papa, V. Venegas. Cryogenics 33, 308 (1993).

[46] P. Mune, E. Govea-Alcaide, R.F. Jardim. Physica C 354, 275 (2001).

[47] Д.А. Балаев, А.А. Дубровский, С.И. Попков, Д.М. Гохфельд, С.В. Семенов, К.А. Шайхутдинов, М.И. Петров. ФTT 54, 11, 11 (2012).

[48] D. López, F. de la Cruz F. Phys. Rev. B 43, 13, 11478 (1991).

[49] D. López, R. Decca, F. de la Cruz. Supercond. Sci. Technol. 5, S276 (1992).

[50] O.V. Gerashchenko, S.L. Ginzburg. Supercond. Sci.Technol. 13, 332 (2000).

[51] A. Kilic, K. Kilic, S. Senoussi, K. Demir. Phys. C 294, 203 (1998).

[52] D. Daghero, P. Mazzetti, A. Stepanescu, P. Tura. Phys. Rev. B 66, 13, 11478 (2002).

[53] D.A. Balaev, A.G. Prus, K.A. Shaykhutdinov, D.M. Gokhfeld, M.I. Petrov. Supercond. Sci. Technol. 20, 495 (2007).

[54] С.В. Семенов, Д.А. Балаев, М.А. Почекутов, Д.А. Великанов. ФТТ 59, 7, 1267 (2017).

[55] D.A. Balaev, S.V. Semenov, M.A. Pochekutov. J. Appl. Phys. 122, 123902 (2017).

[56] A.V. Mitin. Physica C 235-240, 3311 (1994). 
[57] В.В. Деревянко, Т.В. Сухарева, В.А. Финкель. ФТТ 46, 10, 1740 (2004).

[58] В.В. Деревянко, Т.В. Сухарева, В.А. Финкель. ФТТ 49, 10, 1744 (2007).

[59] Т.В. Сухарева, В.А. Финкель. ФТТ 53, 5, 858 (2011).

[60] Д.А. Балаев, А.А. Быков, С.В. Семенов, С.И. Попков, А.А. Дубровский, К.А. Шайхутдинов, М.И. Петров. ФТТ 53, 5, 865 (2011).

[61] Д.А. Балаев, С.В. Семенов, М.И. Петров. ФТТ 55, 12, 2305 (2013).

[62] В.В. Деревянко, Т.В. Сухарева, В.А. Финкель, Ю.Н. Шахов. ФTT 56, 625 (2014).

[63] Д.А. Балаев, Д.М. Гохфельд, А.А. Дубровский, С.И. Попков, К.А. Шайхутдинов, М.И. Петров. ЖЭТФ 132, 1340 (2007).

[64] Д.А. Балаев, А.А. Дубровский, К.А. Шайхутдинов, С.И. Попков, Д.М. Гохфельд, Ю.С. Гохфельд, М.И. Петров. ЖЭТФ 135, 271 (2009).

[65] D.A. Balaev, S.I. Popkov, E.I. Sabitova, S.V. Semenov, K.A. Shaykhutdinov, A.V. Shabanov, M.I. Petrov. J. Appl. Phys. 110, 093918 (2011).

[66] D.A. Balaev, S.V. Semenov, M.I. Petrov. J. Supercond. Nov. Magn. 27, 1425 (2014).

[67] S.V. Semenov, D.A. Balaev. Physica C 550, 19 (2018).

[68] S.V. Semenov, D.A. Balaev. J. Supercond. Nov. Magn. 32, 2409 (2019).

[69] С.В. Семенов, Д.А. Балаев. ФТТ 62, 7, 1008 (2020).

[70] S.V. Semenov, A.D. Balaev, D.A. Balaev. J. Appl. Phys. 125, 033903 (2019).

[71] G. Blatter, M.V. Feigel'man, V.B. Gekshkebein, A.I. Larkin, V.M. Vinokur. Rev. Mod. Phys. 66, 4, 1125 (1994).

[72] M.I. Petrov, D.A. Balaev, K.A. Shaihutdinov, K.S. Aleksandrov. Supercond. Sci. Technol. 14, 798 (2001).

[73] Д.М. Гохфельд. ФТТ 56, 12, 2298 (2014).

[74] Д.М. Гохфельд. Письма ЖТФ 45, 2, 3 (2019).

[75] C.P. Bean. Rev. Mod. Phys. 36, 31 (1964).

[76] C.A.M. dos Santos, M.S. da Luz, B. Ferreira, A.J.S. Machado. Physica C 391, 345 (2003).

[77] D.A. Balaev, S.I. Popkov, S.V. Semenov, A.A. Bykov, E.I. Sabitova, A.A. Dubrovskiy, K.A. Shaikhutdinov, M.I. Petrov. J. Supercond. Nov. Magn. 24, 2129 (2011).

[78] В.В. Деревянко, Т.В. Сухарева, В.А. Финкель. ФТТ 59, 1470 (2017).

[79] D.A. Balaev, S.V. Semenov, D.M. Gokhfeld. J. Supercond. Nov. Magn. (2021). https://doi.org/10.1007/s10948-021-05812-2

[80] U. Gunsenheimer, U. Schussler, R. Kümmel. Phys. Rev. B 49, 6111 (1994).

Редактор Т.Н. Василевская 